\title{
SISTEMAS DE FILTRADO ADAPTATIVO APLICADO AL TRATAMIENTO DIGITAL DEL ELECTROCARDIOGRAMA
}

C el capítulo anterior fueron presentados los sistemas de filtrado digital
tipos FIR. Estos sistemas están basados en un algoritmo que toma un conjunto de muestras de la señal de entrada (en este caso señal ECG) y los opera con un conjunto de coeficientes para generar la muestra de salida. Estos esquemas de filtrado digital son estáticos, lo cual quiere decir que su función de transferencia y respectiva respuesta en frecuencia no cambian con el tiempo. Esto conlleva que las frecuencias que son atenuadas siempre son las mismas. Diversos estudios sobre ruidos que afectan la señal ECG han aportado información que lleva a la conclusión que sus componentes espectrales pueden cambiar con respecto al tiempo. Por lo cual, en condiciones más realistas, los sistemas de filtrado digital FIR no siempre poseen la capacidad de atenuar las distorsiones, ya que no pueden cambiar su respuesta en frecuencia, en caso de un comportamiento dinámico.

Los sistemas de filtrado adaptativo tienen la capacidad de cambiar su función de transferencia, acorde a las señales de entrada y sus propiedades. Estos sistemas son implementados a través de algoritmos que realizan un proceso de actualización de los coeficientes que hacen parte de la estructura interna 
del filtro. Esta actualización permite mejorar la respuesta en frecuencia del sistema de filtrado.

En el presente capítulo se ofrece al lector la oportunidad de obtener una introducción a los sistemas adaptativo de filtrado FIR. El capítulo se centra en el esquema tipo Wiener, el cual requiere una señal de entrada y otra señal de referencia que representa la salida ideal: una versión sin distorsión de la señal de entrada.

\section{Sistema adaptativo basado en un filtro digital FIR}

Un sistema adaptativo basado en un filtro digital FIR se puede representar con un diagrama de bloques, como el de la figura 26. En el diagrama de bloques se puede observar que el sistema adaptativo utiliza como entrada la señal $x[n]$ y la señal de salida y[n]. La señal e[n] corresponde a la señal de error que se genera a partir de la señal y[n] y una señal de referencia $d[n]$.

La señal de referencia puede ser estimada de diversas maneras, por lo general corresponde a una versión de la señal de entrada. Desde el punto de vista matemático, se puede asumir que la señal de entrada equivale a la suma de la señal de referencia y el ruido que se requiere eliminar. FIGURA 26.
DIAGRAMA DE
BLOQUES DE
UN SISTEMA
ADAPTATIVO

FuENTE:

ELABORACIÓN PROPIA.

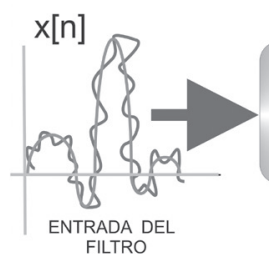

FILTRO
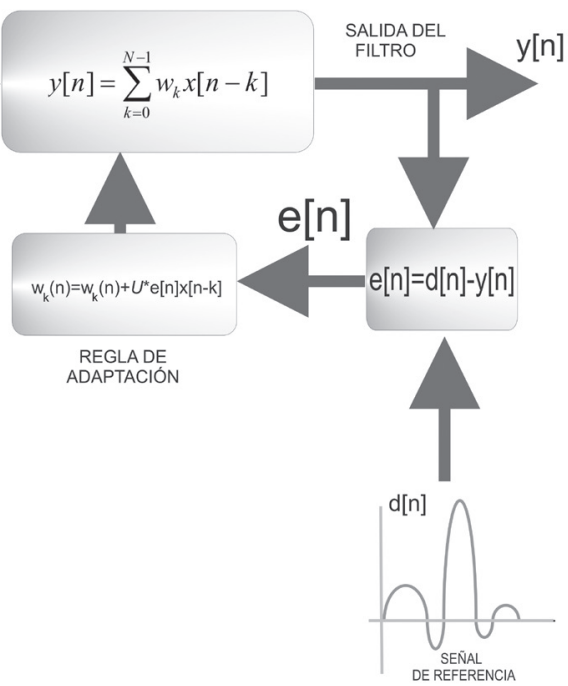
El sistema adaptativo (figura 26) está conformado por un filtro digital convencional FIR. Este filtro parte de una ecuación en diferencia conformada por los pesos $w_{k}$ que son computados con las muestras de la señal de entrada $x[n]$. Ver ecuación 2 .

ECUACIÓN 2.

$$
y[n]=\sum_{k=0}^{N-1} w_{k} x[n-k]
$$

El proceso de filtrado produce la salida $y[n]$ que es comparada con una señal de referencia denominada $\mathrm{d}[\mathrm{n}]$. La diferencia entre $y[n]$ y $\mathrm{d}[\mathrm{n}]$ constituye una señal de error e[n], que es luego llevada a otra etapa que posee una regla de adaptación (ver ecuación 3 ) donde se modifican los coeficientes $w$ del filtro con el objetivo de eliminar el error.

$$
\mathrm{W}(k)=\mathrm{W}(k)+\mu \cdot e[n] . x[n-k]
$$

La regla de adaptación, descrita en la ecuación 3, posee la variable $\mu$ que corresponde al coeficiente de adaptación. Este valor se puede calcular a partir de las muestras de la señal de entrada x(n) [34]. Ver ecuación 4.

ECUACIÓN 4.

$$
\mu=\frac{1}{2 \cdot \sum_{n-0}^{N-1}|x[n]|^{2}}
$$

Los sistemas adaptativos han tenido aplicaciones en el tratamiento digital de la señal ECG. En el caso del ruido de línea de potencia eléctrica, este tipo de filtro representa una alternativa cuando la frecuencia central no corresponde exactamente a $60 \mathrm{~Hz}$ y posee cambios en su valor.

A través de las siguientes líneas de código, se puede simular en Matlab cómo será el comportamiento del escenario planteado con un modelo sinusoidal 
de variación de frecuencia de la señal de tensión de la línea de potencia eléctrica. Este modelo representa un escenario más cercano a la realidad de un ruido, en el cual su componente espectral varía con respecto al tiempo. En el caso de un filtro FIR tipo notch, no sería posible la adecuada atenuación de la respectiva distorsión generada.

A continuación se presenta una propuesta de programa orientado a la simulación de un escenario que visualiza una señal ECG contaminada con un ruido de línea de potencia eléctrica con una situación dinámica. Las líneas de código toman una señal ECG almacenada en la variable $s$ con frecuencia de muestreo Fs y una cantidad de muestras $\mathrm{N}$.

Posteriormente se crea el arreglo de datos de datos frec que representa una función sinusoidal que modela los cambios del valor de la frecuencia central del ruido de línea de potencia eléctrica. La función que modela el cambio de valor de frecuencia tiene un nivel medio con valor de 60 (corresponde a la frecuencia central) y la variación sinusoidal permite que varíe entre 60.9 y 59.9 .

$$
\begin{aligned}
& \text { Ts = 0.008; } \\
& \text { Fs = 1/Ts; } \\
& N=\text { length }(s) ; \\
& n=1: N ; \\
& t=(n-1)^{*} T s ; \\
& \text { frec }=0.9^{*} \cos \left(2^{*} i^{*} 0.05^{*} n^{*} T s\right)+60 ;
\end{aligned}
$$

figure plot(frec)

De manera consecutiva se procede a generar el arreglo de datos $r$ que contiene una señal sinusoidal con un valor de frecuencia central que varía con cada iteración.

$$
\begin{aligned}
& \text { for } n=1: N \\
& r(n)=0.4^{*} \cos \left(2^{*} \operatorname{pi} f r e c(n)^{*} n^{*} T s\right) \text {; } \\
& \text { end } \\
& x=s+r ;
\end{aligned}
$$

La figura 27 ilustra, en el dominio del tiempo y de la frecuencia, la señal ECG distorsionada por un ruido de línea de potencia eléctrica con frecuencia 
variable. En la magnitud del espectro obtenido se puede apreciar la presencia de diferentes picos en la frecuencia de $60 \mathrm{~Hz}$ y en sus valores vecinos. Esta presencia de picos es evidencia del modelo sinusoidal de variación del valor central de la frecuencia del ruido simulado.
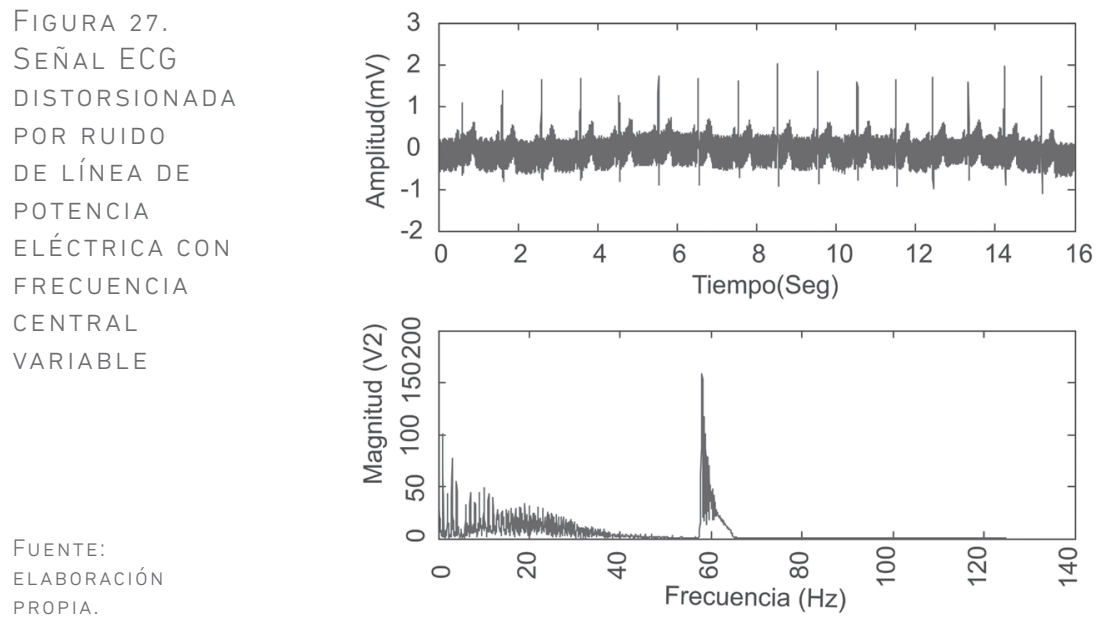

Si este escenario es tratado con un filtro digital FIR tipo muesca, no será posible obtener resultados positivos. Como se puede ver en la figura 24, las componentes espectrales del ruido están dispersas en el espectro de la señal ECG.

Tomando como señal de entrada $x[n]$, la señal electrocardiográfica contaminada con ruido de línea de potencia eléctrica con frecuencia central variable y la variable $d[n]$, como la señal electrocardiográfica libre de distorsiones, se describen a continuación las líneas de código (para el asistente Matlab ${ }^{\circledast}$ ) para el diseño de un filtro rechaza banda por medio de la arquitectura de filtrado adaptativo:

En esta primera etapa del programa propuesto se realiza la inicialización de un arreglo de datos $B$. Este arreglo posee los espacios en memoria de los coeficientes del sistema adaptativo de filtrado. 
\% inicialización de los coeficientes

$$
\begin{aligned}
& B=\operatorname{zeros}(1,60) ; \\
& B(1)=1 ; \\
& L=\text { length }(B) ;
\end{aligned}
$$

En la segunda parte del código se calcula el coeficiente de adaptación, a partir de las muestras de la señal de entrada.

\% Cálculo del paso de coeficiente de $\mu$

Paso $U=1 /\left(2^{*} \operatorname{sum}(x . \wedge 2)\right)$;

La tercera parte del código ejecuta el proceso de adaptación del filtro y se encarga de tomar los valores de los coeficientes y calcular la muestra de la señal de salida. Luego, se calcula el error a través de la resta de la muestra de señal de salida y de la señal de referencia.

$\%$ proceso de adaptación del filtro

$$
\begin{aligned}
& \text { for } n=L+1: N \\
& c=0 ; \\
& \text { for } k=1: L \\
& c=c+B(k) * x(n-k) ; \\
& \text { end } \\
& y(n)=c ; \\
& e(n)=d(n)-y(n) ;
\end{aligned}
$$

\% cálculo de los nuevos valores de coeficientes

$$
\begin{aligned}
& \text { for } k=1: L \\
& B(k)=B(k)+e(n){ }^{*} \text { paso } U^{*} x(n-k) ;
\end{aligned}
$$

end

$\%$ fin de las iteraciones

end

El resultado del proceso de adaptación genera un conjunto de coeficientes que permiten la implementación de un filtro FIR óptimo para solucionar el problema del ruido. La figura 28 ilustra la respuesta en frecuencia del nuevo sistema discreto que está conformado por los coeficientes adaptados. 
FIGURA 28.

RESPUESTA EN

FRECUENCIA

DE UN FILTRO

FIR CON

COEFICIENTES

CALCULADOS A

PARTIR DE UN

PROCESO DE

ADAPTACIÓN

FUENTE:

ELABORACIÓN

PROPIA.

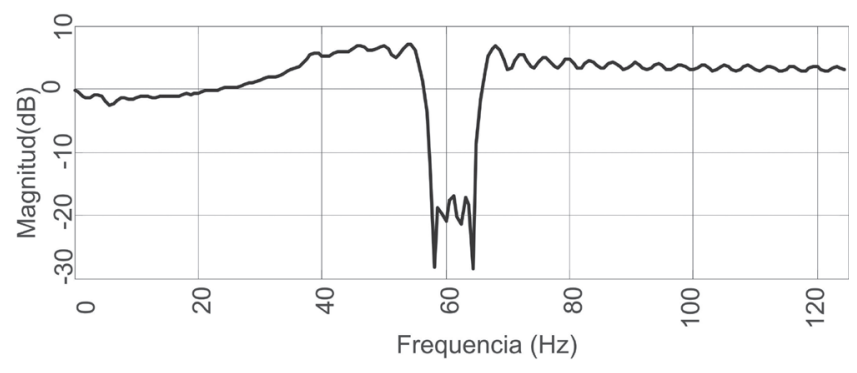

El resultado contenido en la figura 28 ilustra que la respuesta en frecuencia del filtro posee una forma característica, generada por el proceso de adaptación. La banda de rechazo tiene una forma similar acorde con las frecuencias que contiene el ruido simulado. Los coeficientes aportados por el proceso de adaptación pueden ser utilizados para la implementación de un filtro FIR. En caso de que las características del ruido vuelvan a cambiar, los valores de los coeficientes deben ser respectivamente actualizados en un nuevo proceso de adaptación.

El proceso de adaptación puede verse como un método para el diseño de filtros FIR, en el cual no se tiene información exacta de los valores de frecuencia de las componentes que se requieren conservar y las que son requeridas para ser rechazadas. Por lo cual, el resultado final del filtro adaptativo no es generar una señal de salida, es más bien el cálculo de valores de coeficientes para obtener un comportamiento óptimo de filtrado.

Al utilizar los coeficientes calculados a partir del proceso de adaptación se pone a prueba el comportamiento del nuevo filtro digital FIR. La figura 29 contiene el resultado del tratamiento digital de la señal ECG con ruido de línea de potencia eléctrica de frecuencia variable. 
FIGURA 29.

TRATAMIENTO

DE LA SEÑAL

ECG CON UN

FILTRO FIR

COMPUESTO DE

COEFICIENTES

CALCULADOS EN

UN PROCESO DE

ADAPTACIÓN

FUENTE:

ELABORACIÓN

PROPIA.
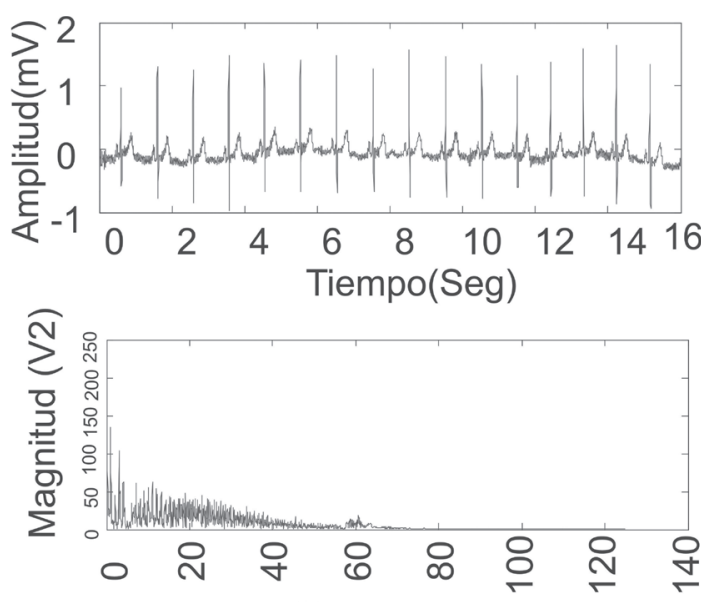

Frecuencia $(\mathrm{Hz})$

\section{Generación de una señal de referencia a partir de regresión polinomial}

En los sistemas de filtrados adaptativos surge el reto del diseño de estrategias para obtener una señal de referencia. El camino sugerido es acudir a la señal de entrada del sistema adaptativo y extraer la señal de referencia a través de una técnica matemática. En el caso del tratamiento digital de la señal ECG, este reto ha sido utilizado para atenuar la distorsión causada por el desplazamiento de la línea de base. Por lo general, este tipo de ruido es generado al interior de sistemas fisiológicos cercanos al cardiovascular, como es el caso del sistema respiratorio. También es incentivado por la actividad muscular generada, por ejemplo, mediante movimientos de brazos y piernas durante la adquisición de la señal ECG. Matemáticamente, se puede afirmar que el desplazamiento de línea de base es de carácter aditivo con respecto a la señal ECG, en cuyo caso es posible modelarlo a través de señales sinusoidales de muy baja frecuencia (inferior a $1 \mathrm{~Hz}$ ).

A continuación se tomarán como objeto de estudio señales ECG con desplazamiento de línea de base y se expondrá, paso a paso, una propuesta de construcción de código en el asistente Matlab para implementar una técnica de estimación del ruido. El primer paso es contar con la señal de estudio y se 
recurre nuevamente a una señal ECG tomada de la base de datos Physionet que está distorsionada por un desplazamiento de su línea de base. La figura 30 ilustra la señal seleccionada.

FIGURA 30.

SEÑAL ECG

TOMADA DE LA

BASE DE DATOS

PHYSIONET CON

DESPLAZAMIENTO

DE LA LÍNEA DE

BASE

FUENTE:

ELABORACIÓN

PROPIA.

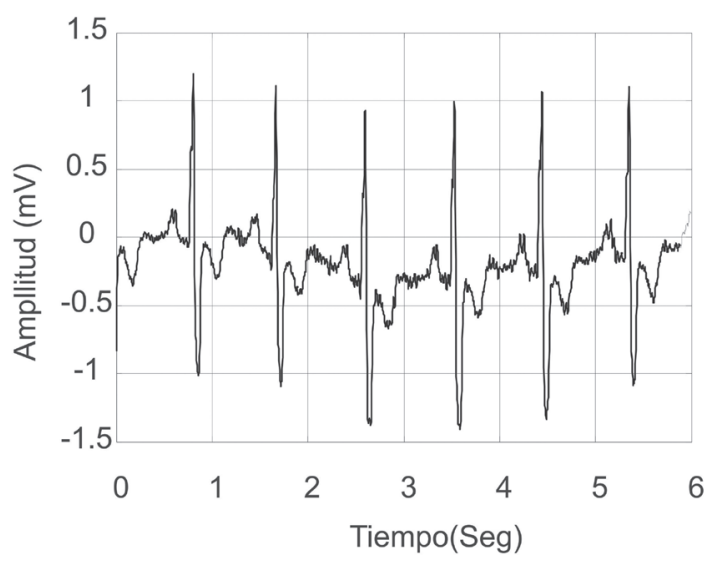

Como se puede ver en la figura 30, el desplazamiento de línea de base es descrito por una curva suave o de muy baja frecuencia. Como método básico para obtener un indicador de este desplazamiento en función del tiempo, se puede optar por el cálculo de valores promedio a lo largo de la señal. Los valores promedios se calculan usando segmentaciones consecutivas de la señal ECG y cada segmento posee un valor de muestras cercano a la duración del complejo QRS. Los valores promedio de la señal de entrada constituyen la materia prima de la señal de referencia [35].

El algoritmo expuesto en las siguientes líneas de código describe el proceso implementado en Matlab $^{\circledR}$. En las siguientes líneas de código es utilizada la variable temporal $c$ para calcular el promedio y proporcionar la variable vma, que contiene los valores promedios calculados para una ventana móvil de 200 muestras. En este caso, el tamaño de la venta fue escogido debido a que el valor de 200 muestras es en promedio la duración del ciclo cardíaco en la señal ECG. 
\% Valores promedio de la señal ECG

\% La señal ECG de entrada está almacenada en la variable p.

$$
\operatorname{vma}(1: 2000)=0 \text {; }
$$

$z=0$;

for $r=1: 10$

$c=0$;

fori $=1: 200$

$c=p(i+z)+c ;$

$x i=c$;

end

$\operatorname{vma}(r)=(1 / 200) * c$

$z=z+200$;

$a(z)=a(r)$;

end

La figura 31 ilustra los datos extraídos de la señal ECG de entrada, almacenados en la variable vma. El resultado obtenido permite verificar que los círculos que corresponden a cada valor de la variable vma indican la variación en el tiempo del desplazamiento de la línea de base.

FIGURA 31. SEÑAL ECG Y VALORES PROMEDIOS CALCULADOS

FUENTE:

ELABORACIÓN PROPIA.

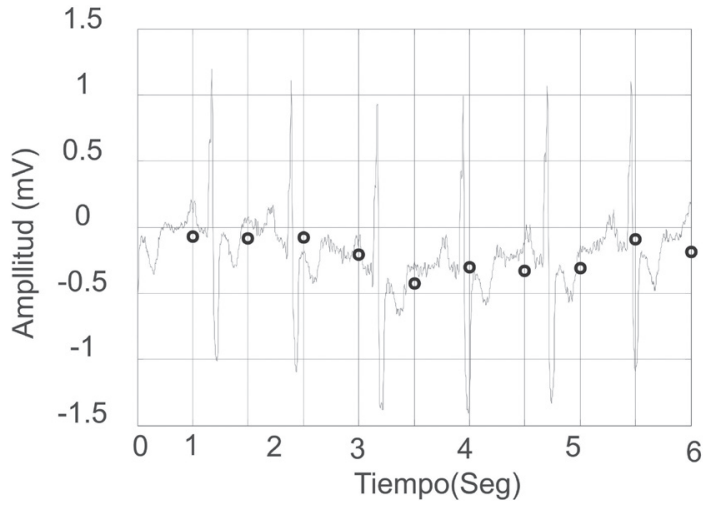


El siguiente paso, en este procedimiento, es el de poder obtener el modelo matemático que describa una curva con la capacidad de unir todos los valores contenidos en la variable vma. A través de técnicas de interpolación polinomial se pueden tomar los datos correspondientes a los valores promedios de la señal ECG para generar una curva que modele el desplazamiento de línea de base. Esta curva se convierte en una función de referencia para ser utilizada en un sistema de cancelación.

Tomando las parejas de datos que corresponden al valor medio y su posición, se utiliza la función interp1 con la finalidad de generar el arreglo de datos $r$, a través de la técnica de interpolación cúbica:

$$
\begin{aligned}
& \text { [p(1)a]; } \\
& n=1: N ; \\
& z=0: 200: N ; \\
& r=\operatorname{interp1}\left(z, a, n,{ }^{\prime} c u b i c '\right) ;
\end{aligned}
$$

Con el fin de mejorar el desempeño del código, se recomienda utilizar como valores iníciales y finales los mismos de la señal ECG original.

La figura 32 ilustra el resultado del proceso de interpolación. Se puede apreciar la curva almacenada en la variable $r$.

FIGURA

32. CURVA

GENERADA A

TRAVÉS DEL

PROCESO DE

INTERPOLACIÓN

CÚBICA
FUENTE:

ELABORACIÓN PROPIA.

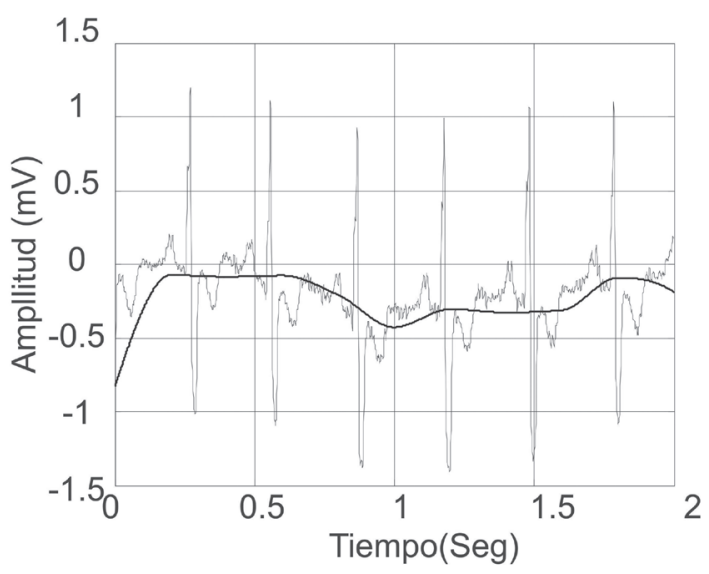


La curva generada en este experimento corresponde a una estimación del modelo del ruido; además se puede asumir que es la curva que se ha adicionado a la señal ECG. Utilizando el diagrama de bloques del sistema de cancelación, expuesto anteriormente en la figura 18, se puede realizar el ensayo para utilizarlo como insumo para el tratamiento del desplazamiento de línea de base.

El proceso de atenuación de la distorsión es ejecutado mediante una resta entre la señal ECG con desplazamiento de línea de base y la función de referencia (figura 33). En este caso, la señal de referencia es obtenida a través de un proceso de interpolación de datos extraídos de la señal de entrada.
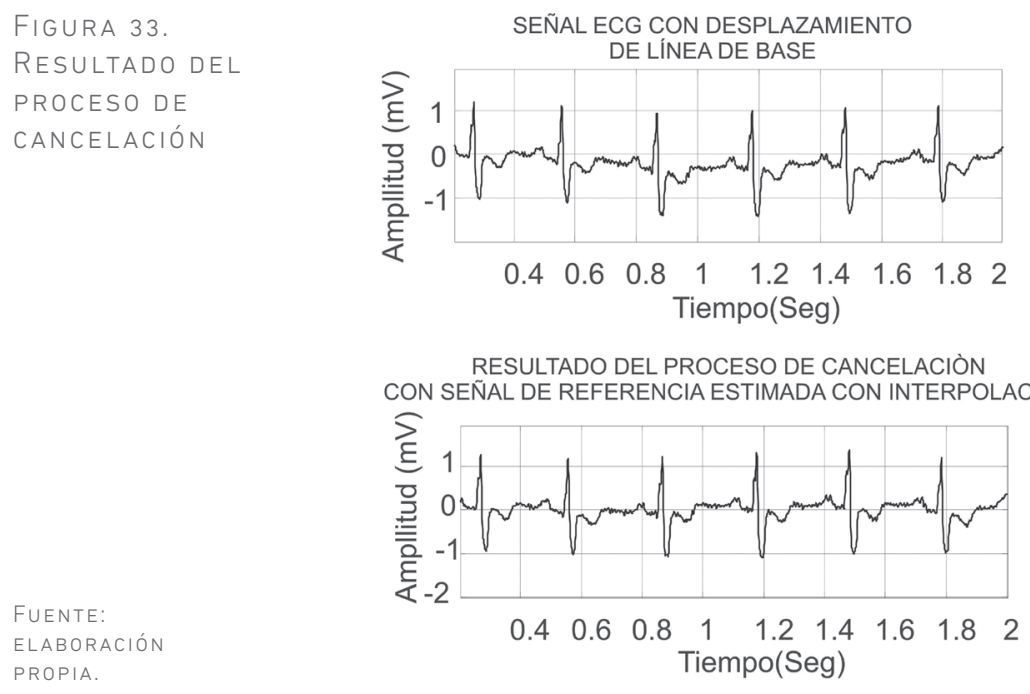

Los sistemas adaptativos representan una alternativa para la remoción de los efectos causados por las distorsiones de línea de potencia eléctrica y desplazamiento de la línea de base. Al atenuar las distorsiones se obtiene un registro electrocardiográfico con gran información contenida en cada una de sus ondas.

Los sistemas adaptativos de filtrado pueden tener diferentes configuraciones, en este caso se han analizado el esquema de Winier y los sistemas de cancelación con extracción de la señal de referencia. 
El esquema de Winier no solo permite filtrar la señal ECG, también es un método para obtener el valor adecuado de los coeficientes del filtro digital con un comportamiento óptimo de respuesta en frecuencia.

El sistema de cancelación también es un esquema de filtrado adaptativo, ya que la señal de referencia cambia de igual manera como lo puede hacer el ruido que causa la distorsión en la señal ECG. 
